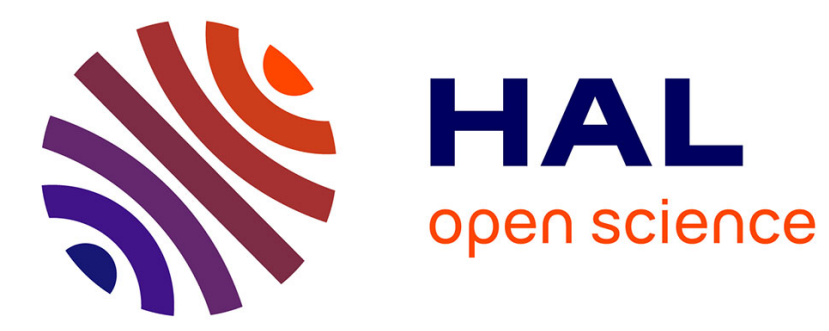

\title{
Le droit et la violence de l'exception. Autour de l'œuvre de Giorgio Agamben
}

Thierry Ménissier

\section{To cite this version:}

Thierry Ménissier. Le droit et la violence de l'exception. Autour de l'œuvre de Giorgio Agamben. Les Cahiers de la justice, 2012. hal-01659720

\section{HAL Id: hal-01659720 \\ https://hal.science/hal-01659720}

Submitted on 8 Dec 2017

HAL is a multi-disciplinary open access archive for the deposit and dissemination of scientific research documents, whether they are published or not. The documents may come from teaching and research institutions in France or abroad, or from public or private research centers.
L'archive ouverte pluridisciplinaire HAL, est destinée au dépôt et à la diffusion de documents scientifiques de niveau recherche, publiés ou non, émanant des établissements d'enseignement et de recherche français ou étrangers, des laboratoires publics ou privés. 


\title{
[ ] DOSSER
}

\section{Le droit et la violence de l'exception. Autour de l'ouvre de Giorgio Agamben}

\author{
par Thierry Ménissier
} Thierry Ménissier, Agrégé de philosophie (1990), docteur de l'École des hautes études en sciences sociales (Centre
Raymond Aron, Paris) en «études politiques » (2000), professeur de philosophie à Grenoble Institut de l'innovation et au
département de philosophie de l'université Pierre Mendès-France - Grenoble 2 .

Cet article entreprend d'abord de restituer les thèses énoncées par Giorgio Agamben dans son ouvrage Homo Sacer, en relevant notamment ce qu'il emprunte à Hannah Arendt, Carl Schmitt, Walter Benjamin et Michel Foucault. En interrogeant la figure énigmatique de l' «homo sacer», et en sondant l'hypothèse de la «vie nue», il s'agit de savoir comment le philosophe italien est conduit à établir que le droit crée de l'exception et qu'il s'exprime originellement par la «mise au ban». Puis l'article se propose d'évaluer une telle construction conceptuelle : la critique agambenienne du droit est-elle fondée et pertinente?

This article first undertakes to present the theses set out by Giorgio Agamben in his work Homo Sacer, pointing out, among other things, his borrowings from Hannah Arendt, Carl Schmitt, Walter Benjamin and Michel Foucault. Looking at the enigmatic "homo sacer" figure and probing the hypothesis of the "bare life", the article explores how the Italian philosopher came to the conclusion that the law creates exception and that this was originally expressed by the "ban from society". The article then proposes to assess this conceptual construction : is the Agambenian criticism of the law valid and relevant?

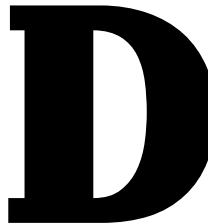

ans les pages qui suivent, nous voulons examiner une discussion qui agite aujourd'hui la philosophie politique, car elle semble susceptible de renouveler l'approche entre, d'une part, les génocides ou violences de masse, et, d'autre part, l'État de droit et, plus généralement, les moyens de juguler ces violences. Cette discussion a pour objet la pertinence et la fécondité de la pensée du philosophe italien Giorgio Agamben (né en
1942, professeur de philosophie esthétique à l'université de Venise jusqu'en 2009). Depuis plus d'une quinzaine d'années, Agamben a construit une interprétation du rapport entre le droit et la barbarie qui apparaît cohérente et suscite la réflexion de par son originalité comme par sa radicalité. Aux juristes, magistrats et philosophes du droit, Agamben adresse même une sorte de défi, dans la mesure où la reconstitution critique qu'il propose du système du droit renverse littéralement les finalités que l'on attend généralement de ce dernier. 
Dossier Le droit et la violence de l'exception. Autour de l'œurre de Giorgio Agamben

\section{L'analyse arendtienne du totalitarisme}

Pour saisir la tentative d'Agamben, il est commode de prendre appui, ainsi qu'il le fait lui-même, sur un certain nombre d'analyses proposées par Hannah Arendt dans son enquête sur la nature, l'origine et la portée du totalitarisme. Si Arendt (1906-1975) n'a nullement inventé l'idée de totalitarisme ${ }^{1}$, elle en fait un usage explicite et l'a indéniablement popularisée. L'auteur des Origines $d u$ totalitarisme ${ }^{2}$ a voulu démontrer que les régimes autoritaires du début du $\mathrm{XX}^{\mathrm{e}}$ siècle, particulièrement le nazisme et le bolchévisme, ne constituent pas seulement des despotismes extrêmes, au sens où Montesquieu expliquait dans De l'esprit des lois que le despotisme est un «gouvernement» reposant sur le «principe» affectif de la crainte $^{3}$, mais qu'ils ont inventé «une nouvelle forme de gouvernement ${ }^{4}$. C'est cette nouvelle forme que le terme de «totalitarisme» entend désigner; quoique cette simple désignation engage en réalité un programme théorique complexe et peut-être impossible à mener complètement à bien. Ainsi que l'explique Bernard Bruneteau dans son ouvrage Les totalitarismes (notons le pluriel), la conceptualisation du totalitarisme est à la fois contingente, nécessaire et

1. Enzo Traverso rappelle que, dès les années 1930, au même moment qu'elle apparaissait dans l'idéologie fasciste, l'idée de "l'État total» a été critiquée par l'opposition au fascisme, d'origine à la fois libérale, chrétienne, socialiste et marxiste. Voir Enzo Traverso, Le totalitarisme. Le $x x^{e}$ siècle en débat, Paris, Éditions du Seuil, 2001.

2. Voir Hannah Arendt, The Origins of Totalitarianism (1re édition, 1951), New York, Harcourt, 1994, trad. Les Origines incertaine ${ }^{5}$. Existe-t-il un type idéal de totalitarisme, nettement caractérisable et accessible à la pensée afin qu'elle puisse agir sur la réalité qu'il désigne? Voilà l'enjeu du problème inauguré notamment par Arendt, et qui n'a pas encore connu de solution. Ce problème touche très exactement à la question des moyens intellectuels dont on dispose pour comprendre les crimes de masse et les prévenir.

Hannah Arendt enquêtait à la fois de manière généalogique, visant à comprendre la naissance du totalitarisme (cette démarche constitue la méthode des deux premiers tomes de son ouvrage, portant respectivement sur «l'antisémitisme» et «l'impérialisme»), et de manière analytique, cherchant à déterminer les éléments qui en composent la notion (démarche sensible surtout dans le troisième tome portant explicitement sur «le totalitarisme»). Des éléments fort variés entrent à vrai dire dans la notion arendtienne de totalitarisme, tels que la transformation d'un antisémitisme culturel en motif d'action politique, l'impérialisme ou le colonialisme des nations européennes reposant sur la dislocation paradoxale de l'Étatnation, l'apparition au sein de ce dernier d'une bureaucratie vectrice d'une idéologie raciste, ou encore la massification des individus de la société libérale. Nous voulons

du totalitarisme, édité sous la direction de P. Bouretz, Paris, Gallimard, «Quarto », 2002.

3. Voir Montesquieu, De l'esprit des lois, Livre I, chapitre 9 «Du principe du gouvernement despotique».

4. Arendt, The Origins of Totalitarianism, op. cit., p. 460-479, trad. p. 813-838.

5. Bernard Bruneteau, Les totalitarismes, Paris, Armand Colin, 1999, chapitres 1, 2 et 3. 
mettre l'accent sur un autre élément: Hannah Arendt souligne le fait que le camp de concentration et d'extermination ne constitue nullement, dans le dispositif totalitaire, une application parmi d'autres de la doctrine sociale et politique. Dans la définition arendtienne, le camp représente plutôt un des principes du système totalitaire: il faut insister sur l'importance du camp en tant que principe d'un système, comme on parle d'un principe dans un système métaphysique ou mathématique ${ }^{6}$ - tel est d'ailleurs une des probables raisons du caractère "fermé » de l'essence du totalitarisme selon Arendt (Cf. le cas si discuté de l'Italie fasciste). Il semble qu'une telle affirmation doive s'entendre en deux sens.

D'une part, le camp gagne à être examiné à l'aune du paradigme de la production; il est toutefois le modèle paradoxal d'une usine contradictoire avec l'idée de production: non seulement, il dépense de manière quasiment somptuaire des moyens que l'économie de guerre rend rares et précieux, mais encore on n'y fabrique rien, littéralement, puisque le type d'«animal humain" dont on envisage la fabrication est contradictoire avec la condition humaine. Celle-ci se nourrit de la pluralité, de la particularité, de la parole mutuelle et de la mémoire collective, autant de choses que le type d'humain animalisé visé par le camp entend réduire à néant. Cependant, Arendt insiste sur la nécessité de concevoir le lien intime qui existe entre

6. Cf. Arendt, The Origins..., op. cit., p. 437-459 : «Total domination », trad. p. 782-812.

7. Hannah Arendt, Between Past and Future. Six Exercises in Political Thought (1re édition 1954), Londres, Penguin Books, le totalitarisme comme dérive étatique du $\mathrm{XX}^{\mathrm{e}}$ siècle et la production de sous-humains dans les camps. D'autre part, le camp joue un rôle capital vis-à-vis de l'application de la doctrine totalitaire, car il manifeste clairement aux yeux de tous, bourreaux comme victimes, que désormais tout est possible. Le camp installe donc les prisonniers dans l'ordre - si l'on ose un tel oxymore - de l'arbitraire pur.

On peut souligner enfin que Hannah Arendt, à l'instar d'autres auteurs de sa génération (tels que Leo Strauss ou Eric Voegelin), s'en est tenue à une sorte d'interrogation inquiète à propos des relations entre la culture moderne et le surgissement du totalitarisme: s'il n'y a pas de liaison nécessaire entre la première et le second, leur liaison semble à la fois réelle ou historique (elle a en effet eu lieu au début du $\mathrm{XX}^{\mathrm{e}}$ siècle lorsque l'Europe s'est suicidée avec la montée du nazisme et du bolchévisme et la guerre mondiale), et toujours possible (Arendt explique à plusieurs reprises dans La Crise de la culture que les éléments du totalitarisme diffusés dans la culture démocratique moderne sont susceptibles de «se cristalliser» à nouveau ${ }^{7}$ ). Arendt, au terme de son enquête, établit donc une double connexion : (1) celle entre le totalitarisme et la production de soushumains dans les camps; (2) celle entre la culture moderne et le surgissement du totalitarisme. La première est nécessaire, la

2006 ; trad. La crise de la culture. Huit exercices de pensée politique, sous la direction de Patrick Lévy, Paris, Gallimard, 1972 ; rééd. «Folio », 1989. 
Dossier Le droit et la violence de l'exception. Autour de l'œurre de Giorgio Agamben

seconde à la fois réelle-historique et possible. En d'autres termes, les États européens conquis par les mouvements autoritaires ont d'une part transformé leur société en systèmes totalitaires qui ont «fabriqué des sous-humains»; et, d'autre part, les démocraties du monde dit libre ne sont pas certaines d'être absolument débarrassées du totalitarisme, car ce dernier est susceptible de survivre aux régimes autoritaires dans les cadres duquel il a initialement été conçu.

\section{Énoncer la condition de l' " homo sacer", un considérable projet éditorial}

C'est cette double connexion qui, reprise et amplifiée, a été interprétée dans un sens original et avec une force singulière par Giorgio Agamben, dans une série de parutions dont la première date de 1995. Il apparaît nécessaire d'identifier comme un seul livre les sept volets actuels d'un ouvrage portant le titre unique de Homo Sacer, l'auteur nous y invite expressément. L'attention à l'ordre de parution des volumes fournit quelques renseignements sur son objet: le premier ${ }^{8}$ dresse les cadres de l'interprétation que nous allons analyser plus bas en plaçant, au centre du propos, la figure de l'homo sacer, issue du droit romain. Et il s'achève par un «seuil» textuel ouvert en direction

8. Homo Sacer. Le pouvoir souverain et la vie nue [Homo Sacer I. Il potere sovrano e la nuda vita, 1995], trad. M. Raiola, Paris, Éditions du Seuil, 1997.

9. Voir Primo Levi, Si c'est un homme (1958), trad. M. Schruoffeneger, Paris, Julliard, 1987, rééd. Pocket, p. 96-97. de l'ouvrage suivant, qui fait référence à la figure du «musulman» [il musulmano; der Muselmann], inventée dans les camps et rapportée par de nombreux auteurs qui ont vécu l'enfer des camps: on en trouve par exemple la mention chez Primo Levi ${ }^{9}$. Le «musulman», écrit Agamben, est «un être chez qui l'humiliation, l'horreur et la peur avaient fini par anéantir toute conscience et toute personnalité, jusqu'à l'apathie (d'où sa dénomination ironique) la plus absolue ${ }^{10}$. Le «musulman» doit donc être regardé comme la «réussite » à la fois la plus désastreuse et la plus complète du projet concentrationnaire, et aussi bien, si l'on réinscrit ce dernier dans la perspective arendtienne, comme la réussite ultime du phénomène totalitaire dans son ensemble : "Muet et absolument seul, il est passé dans un autre monde, sans mémoire et sans compassion ${ }^{11}$. Le second ouvrage publié est présenté comme le troisième volume ${ }^{12}$, et son chapitre 2 traite également, et avec quelle force, du statut du «musulman». Le lecteur est par conséquent tenté de tirer la conclusion qu'homo sacer traite de l'expérience concentrationnaire en entreprenant de déterminer les conditions de dégradation de l'humanité qu'elle a engendrées. En se focalisant sur la figure du «musulman», il consiste en une exploration des postures humaines spéciales ou anormales qui y furent inventées, dont on peut penser

10. Homo Sacer I, trad. p. 199.

11. Ibidem.

12. Ce qui reste d'Auschwitz. L'archive et le témoin. Homo Sacer III [Quel che resta di Auschwitz. L'archivio e il testimone, 1998], trad. P. Alferi, Paris, Rivages, 1999. 
qu'elle est menée dans un esprit proche de la phénoménologie - on sait que Hannah Arendt avait elle-même entrepris une phénoménologie des conditions concentrationnaires, en réfléchissant sur le rapport entre nature et condition humaines ${ }^{13}$. Cette tentation est renforcée par la considération du fait que le dernier volume consiste en une réflexion sur le statut de la littérature de témoignage provenant des camps: il s'agit de comprendre ce que signifient ces textes qui reviennent littéralement de l'enfer, entre le témoignage et l'archive.

Cependant, entre le premier et le troisième volume, l'auteur en a inséré plusieurs autres, qu'il a explicitement désignés comme intermédiaires ${ }^{14}$. La prise en compte de ces volumes fournit d'autres indications sur le projet de l'auteur: les notions d'«état d'exception », de «règne», de «gloire», d'« œuvre de Dieu» qui constituent les objets de ces volumes donnent à penser que le détour, entre la figure théorique, lointaine et mystérieuse, de l'homo sacer et la posture phénoménologique du «musulman » attestée par la littérature contemporaine des camps, est long et ample - si bien que l'ambition du projet s'élève considérablement puisque le propos de l'auteur se com-

13. Cf. Thierry Ménissier, "L'animalité comme limite et comme horizon pour la condition humaine selon Hannah Arendt», dans Jean-Luc Guichet (dir.), Usages politiques de I'animalité, Paris, L'Harmattan, 2008, p. 223-252.

14. Voir Giorgio Agamben, État d'exception. Homo Sacer II, 1 [Lo stato di eccezione. Homo Sacer II, 1, 2003], trad. J. Gayraud, Éditions du Seuil, 2003; Le règne et la gloire. Pour une généalogie théologique de l'économie et du gouvernement. Homo Sacer, II, 2 [II regno e la gloria. Per una genealogia teologica del dell'economia e del governo. Homo Sacer, II, 2, 2007], trad. J. Gayraud et M. Rueff, Paris, Éditions du Seuil, plexifie manifestement d'une théorie de nature juridique (volume II, 1) et s'entend explicitement en regard d'une «théologie politique de l'économie et du gouvernement» (sous-titre du volume II, 2), puis d'une analyse de «l'œuvre de Dieu» (dans le titre du volume II, 5). La trilogie initiale, compliquée de quelques appendices, s'oriente par conséquent dans un registre critique, et plus exactement dans le registre d'une critique philosophique de la modernité menée grâce aux catégories théologiques. Elle se complète plus récemment d'une perspective éthique, encore ouverte à l'heure qu'il est, puisque un seul volume a paru ${ }^{15}$.

Envisagé à présent de manière synthétique, Homo Sacer paraît donc proposer une interprétation du phénomène concentrationnaire - et par suite de l'expérience totalitaire - qui rejaillit sur un ambitieux projet de réflexion des catégories juridiques, éthiques et politiques (bref des catégories normatives) contemporaines. Ainsi restitué dans son ambition, cet ample ouvrage représente un événement intellectuel important même pour ceux qui ne partagent pas les vues d'Agamben. En effet, la littérature actuelle de théorie politique a si bien pris en

2008 ; Le sacrement du langage. Archéologie du sacrement. Homo Sacer, II, 3 [I/ sacramento del linguaggio. Archeologia del sacramento, 2008], trad. J. Gayraud, Paris, Librairie philosophique J. Vrin, 2009; Opus Dei - Archéologie de I'office. Homo Sacer II, 5 [Opus Dei: Archeologia dell'ufficio. Homo Sacer, II, 5, 2012], trad. M. Rueff, Paris, Éditions du Seuil, 2012.

15. Voir Giorgio Agamben, De la très haute pauvreté. Règles et forme de vie. Homo Sacer IV, 1 [Altissima povertà. Regole monastiche e forma di vita. Homo Sacer, IV, I, 2011], trad. J. Gayraud, Paris, Éditions Rivages, 2011. 
Dossier Le droit et la violence de l'exception. Autour de l'œurre de Giorgio Agamben

compte l'ambition intellectuelle de l'ouvrage qu'il est devenu impossible de réfléchir la question de la nature de la souveraineté de l'État moderne et contemporain en incluant l'épisode totalitaire sans accepter la référence à l'interprétation d'Agamben. Que dit cette interprétation?

\section{La critique de la souveraineté}

S'il en reprend certaines conclusions fondamentales, Agamben ajoute à l'enquête arendtienne plusieurs éléments. D'abord, il accepte l'hypothèse de travail émise par Michel Foucault (1926-1984) dans son livre de 1976, La volonté de savoir, et qu'on peut nommer «l'hypothèse biopolitique» ${ }^{16}$. À un certain moment de l'histoire moderne, estime Foucault, les relations du pouvoir et du savoir se sont configurées autrement. Le pouvoir était la capacité de «faire mourir» (et de «laisser vivre»); il est devenu celle de «faire vivre» (et de «laisser mourir»). Le tournant se situe au moment où la vie devient l'objet politique par excellence - en gros à la fin du XVIII e siècle ou dans le courant du XIXe. En mettant l'accent sur une semblable captation de la vie, Foucault entendait se détourner d'une certaine manière de concevoir l'histoire du pouvoir. Il se détourne de la logique juridique de la construction de la souveraineté d'après laquelle on a coutume de penser cette histoire. Dans la perspective ouverte par Foucault, l'histoire du pouvoir

16. Voir Michel Foucault, Histoire de la sexualité, tome I: La volonté de savoir, Paris, Gallimard, 1976, et particulièrement le n'est celle de la capacité souveraine de promouvoir des lois afin de conférer aux individus conçus comme sujets de droits des moyens d'action réglés par la présence réelle ou symbolique de l'État (force de police ou majesté souveraine et autres effets symboliques du pouvoir institutionnel) - pour reprendre la caractérisation de la souveraineté dans les termes fondateurs de Jean Bodin dans les Six Livres de la République (1576). Il s'agit désormais de concevoir comme furent prises en charge, de manière en grande partie invisible, les conditions de la vie naturelle et de quelle manière furent gérées les conditions d'existence des populations.

Agamben prend appui sur cette hypothèse dans le but de se livrer à une opération complexe qui, me semble-t-il, coordonne trois niveaux. Il s'agit en effet pour lui (1) de mener à bien une réinterprétation de la notion de souveraineté et de son histoire, tandis que, en s'engageant dans l'hypothèse biopolitique, Foucault s'était purement et simplement détourné de cette notion; (2) d'engager une relecture sur de nouvelles bases de toute la tradition de philosophie politique moderne, en particulier une relecture de la signification et de la portée des arguments développés, de manière fondatrice, par le philosophe anglais Thomas Hobbes notamment dans le Léviathan de 1651 ; (3) de donner à voir de manière originale les relations entre l'idée «normale» de l'État moderne et les massacres, géno-

chapitre V, «Droit de mort et pouvoir sur la vie», «Tel», p. 177-211. 
cides et formes de domination extrêmes que connaît l'époque contemporaine.

En d'autres termes, on peut affirmer que l'État moderne est devenu fort tôt - puisque une telle évolution est sensible dès le XVII ${ }^{\text {e }}$ siècle, estime Agamben - un des moyens privilégiés de la biopolitique. De la sorte, en dépit des apparences, la philosophie politique moderne prépare tout autre chose que l'émancipation de libres subjectivités, mais plutôt la domination biopolitique des corps ainsi que la gestion des populations - elle n'œuvre pas à la constitution du socle métaphysique ni des cadres juridiques de la société démocratique, ou bien il convient de réinterpréter la signification de cette dernière. Ce double contexte, pratique et théorique, conduit à l'idée que l'apparition historique des totalitarismes doit elle aussi être reconsidérée: l'émergence de tels régimes monstrueux ne représente nullement un phénomène contingent mais constitue une des expressions possibles et finalement prévisibles de la nature biopolitique de la souveraineté, en partie masquée par les dénégations ou les contre-feux de la philosophie politique classique.

Afin d'étayer son interprétation, Agamben ajoute à l'hypothèse foucaldienne un élément théorique de toute première importance, à savoir une caractérisation de la souveraineté qui emprunte à Carl Schmitt - le juriste qui fut un des soutiens du régime nazi en matière de théorie du droit (1888-
1985) - l'idée que l'exception en est la catégorie centrale de l'ordre juridique. Cet ajout s'agrémente d'une originalité théorique intéressante: Agamben entremêle les considérations schmittiennes et les spéculations d'un auteur qu'il est normalement impossible de conjuguer avec le juriste nazi: l'écrivain Walter Benjamin. Ce dernier (1892-1940), philosophe et historien de l'art, ami d'Hannah Arendt, était lié à l'École de Francfort, soit aux auteurs de la «théorie critique» d'obédience marxiste tels que Theodor Adorno et Max Horkheimer, et connut une mort tragique puisqu'il se suicida à la frontière franco-espagnole en tentant de fuir le nazisme. Or, Benjamin avait, dans des pages étonnantes par leur acuité métaphysique, esquissé une puissante réflexion sur l'origine et la nature de la violence, en particulier dans un texte important de $1921^{17}$. Dans cette analyse, notamment, le droit se voit confronté à sa propre limite, car il est ramené à la contradiction existant entre son origine et les fins qu'il poursuit. La première apparaît injustifiable en dernière analyse : le système juridique que se donne une nation ne saurait se justifier d'une justice intrinsèque, il semble toujours relatif à des conditions historiques et à la défense d'une communauté particulière. Les secondes, en revanche, semblent rationnellement et éthiquement justifiées, même s'il s'agit d'une construction a posteriori ou d'une rétrospective. Dans une page d'un très haut niveau

17. Voir Walter Benjamin, "Critique de la violence » [1921], trad. M. de Gandillac, revue par R. Rochlitz, dans Fuvres, I, Paris, Gallimard, Folio, 2000, p. 210-243. 
Dossier Le droit et la violence de l'exception. Autour de l'œurre de Giorgio Agamben

analytique, Benjamin expose ce qu'il nomme «la singulière expérience, à première vue décourageante, qui correspond au caractère finalement indécidable de tous les problèmes de droit (expérience qui, dans son absence de toute issue possible, n'a peut-être de comparable que l'impossibilité où se trouvent toutes les langues naissantes de décider de quelque façon concluante sur le «correct» ou le «faux») » ${ }^{18}$. Enfin, il déduit de la limite intrinsèque de tout droit l'idée d'un fondement «mythique» ou «divin» de la violence d'État. Ce dépassement mythologique ou théologique donne à penser à Agamben que les questions posées par le rapport entre le droit et sa justification dans la réalité (1) dépassent le régime normal de la rationalité, (2) impliquent un ordre de faits excédant fondamentalement le plan juridique, un ordre de faits qui relèvent de la métaphysique ou de l'esthétique, lesquelles confèrent aux règles de droit le sens qui leur fait initialement défaut et également, de ce fait, leur valeur opératoire.

\section{Le droit créateur de «l'état d'exception » ?}

Si l'on recentre à présent l'analyse sur le problème qu'Agamben hérite de Schmitt, il convient de reconnaître la difficulté devant

18. Ibidem, p. 233.

19. Carl Schmitt, Théologie politique [1922], trad. J.-L. Schlegel, Paris, Gallimard, 1988. Voir également Le Léviathan dans la doctrine de l'État de Thomas Hobbes. Sens et échec d'un symbole politique [1938], trad. M. Köller et D. Séglard, Paris, Éditions du Seuil, 2002. Sur le droit constitutionnel selon Carl laquelle le philosophe italien se trouve placé : concilier la genèse de l'ordre juridique régulier et la centralité de la catégorie d'exception relève évidemment d'une véritable gageure, tant ces termes semblent exclusifs l'un de l'autre. Le volume II, 1 de Homo Sacer est précisément consacré à cette épineuse question, tant au niveau de l'analyse des textes qu'à celui de l'examen des pratiques juridiques les plus diverses. Afin de réduire le hiatus entre la norme et l'exception, Agamben en appelle notamment à la thèse centrale du décisionnisme schmittien : il pointe le caractère dynamique de l'acte d'institution du droit, puisque est défini comme souverain celui qui «décide de la situation d'exception » pour reprendre la fameuse expression de la Théologie politique de Schmitt ${ }^{19}$. Selon Agamben, il est nécessaire de repenser les éléments de la théorie foucaldienne de la biopolitique (le pouvoir est pouvoir sur la vie) à la lumière de l'acte d'institution selon Schmitt; ainsi «la production d'un corps biopolitique est l'acte original du pouvoir souverain» ${ }^{20}$. Ici, c'est la notion d'acte qui est centrale. Et son impact est très grand, car elle réorganise considérablement les intuitions foucaldiennes. Surgit en effet une conséquence importante: si, dans les études menées par Foucault, la biopolitique obéissait à des règles de discipline des individus et de gestion des populations, dans celles qu'entreprend Agamben les déci-

Schmitt, voir l'excellente étude de Sandrine Baume, Carl Schmitt, penseur de l'État. Genèse d'une doctrine, Paris, Presses de la Fondation nationale des sciences politiques, 2008.

20. Homo Sacer I, introduction, trad. p. 14, souligné par l'auteur. 
sions de faire exception et d'exclure jouent un rôle cardinal. Une telle modification est susceptible de s'entendre de trois manières différentes.

Premièrement, la forme du ban (le bannissement) se profile comme la structure première de la souveraineté: celle-ci consiste moins à inclure qu'à exclure - tel est l'enjeu herméneutique de fond de la relecture de Hobbes par Agamben, pour qui il s'agit de montrer que Hobbes lui-même était bien conscient de la nature de la souveraineté au moment où il en forgeait le type pour toute la modernité rationaliste ${ }^{21}$.

Deuxièmement, à l'époque contemporaine, le camp donne à penser sur la souveraineté entendue comme création de l'exception car "le camp est l'espace qui s'ouvre lorsque l'état d'exception commence à devenir la règle » 22 ; par conséquent, le camp doit être regardé avec toute l'étrangeté qu'il réclame : «il est un morceau de territoire situé en dehors de l'ordre juridique normal, mais il n'est pas pour autant un simple espace extérieur ». À noter cette remarque qui, introduite ici, prend un relief particulier - un fait connu des spécialistes d'histoire du droit, mais finalement peu considérée par la philosophie politique avant la remarque d'Agamben: la matrice du dispositif juridique de l'état d'exception promue entre autres par un Carl Schmitt dans un contexte nazi se trouvait déjà dans la constitution de la République de Weimar. Une telle

21. Luc Foisneau, "Souveraineté et animalité. Agamben lecteur de Hobbes », dans Thierry Gontier (dir.), Animal et animalité dans la philosophie à la Renaissance et à l'âge classique, Louvain-Paris, Peeters, 2005, p 231-244. remarque offre à l'auteur italien le moyen de suggérer que la mise en œuvre légale de l'exception n'a pas été le seul fait de la perversion générale qu'est le nazisme; si bien que de son point de vue le camp, faisant culminer l'instauration de l'exception dans ce qui malgré tout apparaît comme un ensemble de normes, n'est que la concrétisation d'une tendance souterraine parcourant l'époque contemporaine. De sorte que, puisque l'état d'exception y est «voulu», puisque celui-ci s'y trouve "capturé dans l'ordre juridique », le camp «inaugure un nouveau paradigme juridico-politique dans lequel la norme devient indiscernable de l'exception» ${ }^{23}$.

Troisièmement, il convient de prendre la mesure du modèle de l'homo sacer, termes avec lesquels Agamben évoque une figure du droit romain repérée depuis longtemps par les savants et pourtant énigmatique dès l'Antiquité: ce statut de l'«homme sacré» est celui de quelqu'un qui se trouve condamné à une existence dégradée car ne pouvant être sacrifiée aux dieux, quoique pouvant être anéantie par n'importe qui, le meurtrier bénéficiant en pareil cas d'une totale impunité. Avec l'homo sacer, il se produit comme une suspension dans le système normal des règles et des obligations que s'impose la société romaine, son statut renvoie à une vacance juridique - à une forme d'état d'exception intégré dans ce qui, par son existence même, conjure l'état d'excep-

22. Homo Sacer I, p. 182, souligné par l'auteur. 23. Ibidem, p. 183 
Dossier Le droit et la violence de l'exception. Autour de l'œurre de Giorgio Agamben

tion, à savoir le système du droit. Une telle posture, d'abord étudiée par l'auteur dans son contexte d'origine du droit romain, est adaptée par Agamben à son propos comme étant celle de la «vie nue» (en italien: la nuda vita); l'ouvrage suggère que cette disposition, il est permis de l'appliquer à des postures très différentes que peut prendre l'existence humaine dans la société contemporaine. Agamben entend avec elle caractériser du point de vue phénoménologique des situations à la fois anormales dans nos sociétés traversées par le droit et récurrentes - et de ce fait, dans le fond, impensées. Agamben en vient à proposer l'idée que, dans leur principe, les sociétés dites démocratiques ne sont pas moins régies par une souveraineté d'exception de caractère biopolitique où s'expérimentent les formes de «vie nue» que ne l'étaient les régimes totalitaires, voire les camps de concentration. C'est là un des aspects les plus provocants de son argumentation: l'auteur italien réunit en une même famille, avec des différences ne portant que sur le degré de violence manifesté, ce qui s'est passé dans les camps totalitaires nazis, et ce qui se passe aujourd'hui dans des lieux tels que Guantanamo, mais aussi Sangatte, ou encore dans les zones de transit des aéroports internationaux. Les camps non moins que la logique d'État biopolitique créent de l'insécurité et des zones de non droit, en tout cas de l'exclusion par rapport à l'humanité décente ou normale, entendue comme la condition d'animal politique dont parlait déjà Aristote. Le camp nous donne un aperçu d'une forme de vie le plus souvent cachée, et même refoulée, par les construc- tions philosophiques et juridiques, aussi bien que par les discours des responsables publics, mais si présente ou récurrente qu'elle apparaît, finalement, comme typique de l'emprise de l'État dans les sociétés modernes.

Non que le citoyen des sociétés démocratiques connaisse la condition du «musulman »; mais cette dernière, estime Agamben, constitue tout de même la forme extrême de l'existence sous le gouvernement biopolitique de ces sociétés, et en tant que telle elle renvoie bien aux formes de «vie nue » qu'y connaissent les individus les plus exposés au pouvoir d'État. La relation entre l'homo sacer des Romains, le «musulman» des camps de concentration et l'individu soumis à la biopolitique moderne et contemporaine s'éclaire en faisant la conjecture suivante: dans le premier cas, si l'on suit le raisonnement d'Agamben, on a affaire au statut de quelqu'un qui a perdu toute dignité au sens ancien de la dignitas: il n'occupe aucun rang social identifiable, il est même nié comme être humain socialisé, tel un paria sans aucun statut (stricto sensu, c'est donc une condition pire que celle d'un paria); dans le deuxième cas, il s'agit d'un être humain dont la volonté est brisée, qui ne peut plus résister par ses forces, car pour lui la loi du camp, aussi cruelle qu'absurde, est devenue sa propre loi - ce qui est précisément impossible pour un humain normal; dans le troisième cas, un individu précarisé en ce qui concerne la gestion de son existence du fait des politiques publiques que l'État exerce sur le corps des populations, selon un spectre de mesures allant de l'hygiène élémentaire à l'éducation et au tra- 
vail. Quel est le point commun entre ces trois situations? Il semble pouvoir être décelé dans le rapport à la mort: tous les trois sont dépossédés de leur mort, ou susceptibles de l'être d'une manière ou d'une autre. Du fait de son statut juridique paradoxal, l'homo sacer est en effet fondamentalement dépossédable de sa mort, et dans les faits il peut l'être à tout moment par l'homme qui a décidé de le tuer et dont on considère qu'il ne commet par là aucune faute juridique; le musulman connaît une situation comparable parce qu'il n'est plus qu'une chose, un «cadavre vivant», dont la mort «industrielle » n'est pas une mort au sens humain du terme ${ }^{24}$; enfin, le corps biopolitique est dépossédé de sa mort par un pouvoir qui, s'il «fait vivre et laisse mourir», est aussi capable, pour gérer les populations, de les «faire mourir» - cependant que ce pouvoir en médicalisant la mort l'a soustraite au contrôle direct du sujet sur lui-même en la soumettant au pouvoir sur la vie, et l'a rendue également insignifiante, en l'excluant des rites sociaux ${ }^{25}$. Qui plus est, comme l'État est le seul agent producteur du droit (on reconnaît ici l'hypothèse décisionniste schmittienne), il expose les justiciables qui dépendent de lui à une forme subtile de «vie nue». Par conséquent, ce rapprochement effectué par Agamben entre, d'une part, les deux extraordinaires figures d'humanité que sont l'homo sacer et le musulman et les modes biopolitiques des sociétés contemporaines, d'autre part, signi-

24. Voir les remarques d'Agamben dans Ce qui reste d'Auschwitz, p. 59-89. fie que l'État moderne ou de droit ne préserve de rien, tout au contraire: exposés à un pouvoir toujours capable de "dénuder la vie », et de multiples manières inouïes, tous les citoyens sont des «musulmans» en puissance.

\section{Quel jugement porter sur la construction théorique d'Agamben?}

Nous disposons à présent de suffisamment d'éléments pour porter un jugement sur l'interprétation que Giorgio Agamben, à partir des intuitions arendtiennes à propos du lien entre l'histoire de la souveraineté et la «production» concentrationnaire, a donné de la relation entre droit, exclusion et barbarie. Ce jugement gagne à être nuancé.

Il se décline en premier lieu à partir de la reconnaissance d'une certaine pertinence, puisque l'interprétation d'Agamben nous donne à réfléchir sur une histoire, la nôtre, au sein de laquelle le surgissement historique des camps de concentration et des massacres étatiques de masse, loin de se réduire à l'expression circonstancielle de politiques particulièrement anormales, peut également être envisagé avec fruit selon la longue histoire et en fonction de causes structurelles typiques de la modernité. Indiscutablement, le philosophe italien nous fournit des moyens intellectuels pour reconsidérer le

25. Voir ibid., p. 89-91, l'interprétation qu'Agamben donne de ce point chez Foucault. 
Dossier Le droit et la violence de l'exception. Autour de l'œurre de Giorgio Agamben

lien entre l'histoire moderne et ce surgissement. Dans un tel contexte et du point de vue de l'hypothèse d'Agamben, il n'y a en réalité aucune énigme en soi dans le surgissement de la production rationnelle de la mort. De ce fait, Agamben nous assigne en quelque sorte à notre propre histoire. Sa tentative prend rang parmi celles d'autres auteurs, on peut penser par exemple à des constructions issues de penseurs libéraux et aujourd'hui classiques telles que celle autrefois déployée dans l'ouvrage de Jacob Talmon, Les Origines de la démocratie totalitaire ${ }^{26}$, ou aux essais qu'Isaïah Berlin a consacrés aux origines du totalitarisme chez Joseph de Maistre ${ }^{27}$ ou au détournement de la liberté par les Lumières, dont Rousseau ou Helvétius seraient responsables ${ }^{28}$. À ces considérations, on peut ajouter la remarque suivante: composé durant la première moitié de la décennie 1990, le premier volume d'Homo Sacer est contemporain des épisodes dramatiques de la guerre dans les Balkans, soit lorsque les Européens furent obligés de reconnaître le fait que la barbarie pouvait renaître en Europe et que le droit se trouvait singulièrement démuni pour lui répondre; l'entreprise éditoriale s'est poursuivie dans la période où, pour répondre à la terreur sourde qui les menaçait, les États-Unis ont effectivement proposé des mesures politiques et juridiques d'exception. Telle est la seconde

26. Voir Jacob Talmonn, Les Origines de la démocratie totalitaire (1952), trad. P. Fara, Paris, Calmann-Lévy, 1966.

27. Voir Isaïah Berlin, "Joseph de Maistre et les origines du totalitarisme ", in Le Bois tordu de I'humanité: Romantisme, nationalisme, totalitarisme (1990), trad. M. Thymbres, Albin Michel, 1992. raison qui nous incite à lire Agamben: la pertinence de sa thèse se mesure avant tout à la qualité de l'analyse grâce à laquelle il indique le caractère hautement instable du système normal de l'État de droit en des temps agités. À cet égard, les ouvrages du philosophe italien gagnent à être lus comme une méditation approfondie sur les métamorphoses de la puissance ${ }^{29}$. On peut ajouter que, s'il existe une certaine valeur éthique de l'inquiétude, cette dernière devient quasiment une vertu politique grâce aux ouvrages d'Agamben car ils nous rappellent la fragilité du lien qui sépare la situation de calme social et celle où règne la barbarie.

Cependant, en second lieu, notre jugement peut être davantage réservé. Moyennant le détour par la thèse foucaldienne de l'essor du biopouvoir, amplifié par la construction schmittienne portant sur l'institution juridique de l'exception et par les réflexions métaphysiques de Benjamin sur la violence, pour Agamben l'État de droit n'est pas du tout par lui-même un dispositif capable de garantir contre les massacres de masse; il est même toujours susceptible de produire de l'exception et d'organiser biopolitiquement la gestion de populations disciplinées, ces deux modalités dont le camp n'est en fait que le passage à l'extrême. Pour le dire avec la force des mots employés par

28. Voir Isaïah Berlin, La liberté et ses traîtres : six ennemis de la liberté, trad. L. Follio, Paris, Éditions Payot \& Rivages, 2007. 29. Voir pour une interprétation en ce sens, Saverio Ansaldi, «Biopolitique, état d'exception, puissance: note sur une politique à venir (autour de Giorgio Agamben)», Revue philosophique de la France et de l'étranger, 2010/3 tome 135, p. 381394. 
Agamben, tandis que la cité (la polis des auteurs grecs) constituait le paradigme du pouvoir classique, le camp (construction biopolitique par excellence) est devenu le paradigme du pouvoir contemporain ${ }^{30}$. Or, une telle vue peut être contestée, tant avec les ressources de la science politique qu'avec celles de la philosophie politique: si cette approche permet de revenir sur le caractère choquant de l'assimilation du droit régulier à l'état d'exception, il s'agit ici également de revenir sur le travail qu'Agamben fait subir à ses sources arendtiennes et foucaldiennes ${ }^{31}$. De surcroît, les sociétés démocratiques, si on les étudie du point de vue de la première, ne recèlent-elles pas certains dispositifs capables d'arrêter la tentation biopolitique de l'État? Et ne dispose-t-on pas, avec les capacités inventives de la seconde, de moyens d'imagination et d'action collectives propres à redonner prise aux citoyens sur leurs destinées communes? Pour le dire en une formule finale: tandis que la pertinence critique de l'interprétation d'Agamben paraît grande, sa portée normative me semble nettement plus faible. Mais, évidemment, la première dimension est amenée à éclairer la seconde: si l'on veut repenser la dimension politique de la société démocratique, quel meilleur avertissement à propos de la violence de masse que celui délivré par le philosophe italien? Reste à construire les éléments de théorie normative dont la

30. Cf. le titre du dernier chapitre de Homo Sacer I: "Le camp comme nomos de la modernité »; le nomos est la loi fondamentale de constitution d'un ensemble.

31. Voir, pour une tentative en ce sens, la belle étude de Martine Leibovici, «Biopolitique et compréhension du totalita- société démocratique et l'agir commun républicain ont besoin. Non seulement il n'y a aucune fatalité dans la captation biopolitique de la politique contemporaine, mais on veut parier sur des modes politiques qui libèrent l'homme au lieu de l'asservir.

À cet égard, il est fortement tentant d'essayer d'aller avec Agamben là où Agamben ne va pas. Soit par exemple ces deux pistes, théoriques et pratiques. Premièrement, si l'état d'exception est une des conditions de possibilité de l'exclusion et de la violence, sourde ou explicite, faite à la population d'un État ou à des groupes ethniques, culturels ou religieux, ne pourrait-on pas demander aux juristes du droit public de se prononcer s'il est ou non possible de limiter, voire de conjurer, l'état d'exception ? ${ }^{32}$ Deuxièmement, comme l'État joue de l'exclusion, ainsi que le montre Agamben, il faut se donner pour tâche de philosophie politique de revenir sans relâche sur les critères d'attribution de la citoyenneté, puisque le droit de cité détermine à la fois protection des individus par l'État et - malgré tout - la capacité d'action des citoyens sur l'État. Cette tâche ouvre ensuite la voie pour une redéfinition des critères de l'activité ou des prérogatives des citoyens dans l'espace public.

risme. Foucault, Agamben, Arendt», in Tumultes, 2005/2, $n^{\circ} 25$, p. 23-45.

32. Voir, à ce propos, le récent dossier de la revue Tracés, n 20-2011/1, «Politiques de l'exception », et particulièrement I'article de Mathieu Carpentier, «État d'exception et dictature », p. 75-93. 
Dossier Le droit et la violence de l'exception. Autour de l'œurre de Giorgio Agamben

\section{Présentation de l'auteur:}

Thierry Ménissier, né en 1964, agrégé de philosophie (1990), docteur de l'École des hautes études en sciences sociales (Centre Raymond Aron, Paris) en «études politiques» (2000), habilité à diriger les recherches en science politique (IEP Grenoble, 2008). Professeur de philosophie à Grenoble, Institut de l'innovation et au département de philosophie de l'université Pierre Mendès-France - Grenoble 2; cher- cheur dans l'équipe Philosophie, Langages $\mathcal{E}$ Cognition, responsable dans cette équipe du réseau RePFI (Recherches philosophiques franco-italiennes); participe aux travaux consacrés à l'innovation dans le cadre de Grenoble Institut de l'innovation et de la structure fédérative de recherche Innovacs (Innovation, Connaissance, Société). Dernier ouvrage paru: La Liberté des contemporains. Pourquoi il faut rénover la République, Grenoble, PUG, 2011. 